\title{
Understanding pulmonary cystic fibrosis
}

\section{Editorial}

Cystic fibrosis, an inherited disease that causes sticky, thick mucus to form in the lungs, pancreas, and other organs, but $90 \%$ of cases affected the airways. More than 10million Americans carry the cystic fibrosis gene without recognizing it. Approximately 1,000 new cases of cystic fibrosis are diagnosed each year and about 30,000 American people sick with cystic fibrosis, particularly, the Caucasians of Northern European descent (estimated 1 in 2,500 Caucasian births). The Asian Americans and native Hawaiians/Pacific islanders are found that 1 in 31,000 to more than 1 in 100,000 Asian American births are affected by cystic fibrosis. Currently, almost $45 \%$ of the cystic fibrosis population is aged 18years or older. Patients with cystic fibrosis are at increased risk of osteoporosis, male infertility, and diabetes. Respiratory failure is the most common cause of death among patients with cystic fibrosis. The age-adjusted death rate among the Caucasians $(0.22$ per 100,000$)$ is much higher than that among other racial and ethnic groups. Half of the patients had the median age of survival of 37.4years in 2008, up from 32years in 2000. When two carriers of the defective gene conceive, there is a $50 \%$ chance that child will inherit one defective gene and be a carrier, but not have cystic fibrosis and $25 \%$ chance that their child will have cystic fibrosis.

The genetic basis for cystic fibrosis is well-characterized, severe monogenic recessive disorder, that arises from mutations in the cystic fibrosis trans membrane conductance regulator $(C F T R)$ gene. There are several molecules that interact with $C F T R$ gene, such as ORCC, ENaC, ROMK2, Aquaporin 3 (AQP3), NKCC1, NBC-1, Syntaxin $1 \mathrm{~A}$, and NHERF/EBP50. The ORCC molecule outwardly rectifies the chloride channel. Since 1989, more than 1,500 specific gene mutations and DNA sequence variations have been identified in CFTR gene. The Delta F508 mutation is the most common and is identified in almost all racial and ethnic groups (approximately $70 \%$ of all cystic fibrosis genes). The sweat ducts of the patients are impermeable to the chloride ions, thus, the sodium chloride remains in the secretions contributing the salty sweat. Typical symptoms includes salty-tasting skin, wheezing or shortness of breath, frequent lung and par nasal sinus infections, nasal polyps, poor growth and poor weight gain, swollen belly accompanied by abdominal gas and discomfort, and broadening of the fingertips and toes. A sweat chloride concentration of more than $60 \mathrm{mmol} /$ liter determined on two or more occasions by quantitative pilocarpineionophoresis remains the "gold standard" for diagnosis, but false-negative sweat tests can also be a consequence of the particular combination of CFTR mutations carried by an individual cystic fibrosis patient. The genetic testing is available, but is not commonly used because it cannot detect all gene mutations (approximately $70-75 \%$ of the detection rate).

\author{
Volume 2 Issue 4 - 2015
}

\author{
Attapon Cheepsattayakorn, ${ }^{1,2}$ Ruangrong \\ Cheepsattayakorn $^{3}$ \\ 'IOth Zonal Tuberculosis and Chest Disease Center, \\ Department of Disease Control, Thailand \\ ${ }^{2}$ IOth Office of Disease Prevention and Control, Department of \\ Disease Control,Thailand \\ ${ }^{3}$ Department of Pathology, Faculty of Medicine, Chiang Mai \\ University, Thailand
}

Correspondence: Attapon Cheepsattayakorn, I0th Zonal

Tuberculosis and Chest Disease center, 143 Sridornchai Road,

Changklan Muang, Chiang Mai 50100, Thailand, Tel 6653 I40767, 6653 276364, Fax 6653 |40773, 6653 273590,

Email attaponche@yahoo.com

Received: April 10,2015 | Published: April 13,2015

Diagnosis of cystic fibrosis is confirmed by directly measurement of the CFTR function. Gene therapy is possible, which the goal is for the DNA to produce a protein needed by patients with cystic fibrosis to correct the basic defect of cystic fibrosis cells. A genetically engineered enzyme called recombinant human deoxyribonuclease (rhDNase), or simply DNase, is administered in aerosol form. This drug thins out the mucus that clogs the patients' airways, reducing the number of lung infections, and improving the lung function. This drug is used in addition to the standard therapies. Currently, gene therapy is deemed safe and effective by the medical community, but it will not be available. Over the past twenty years, double lung transplantation has replaced heart-lung transplantation, but lacking donors and having long waiting list. The common treatments for those with cystic fibrosis include airway clearance techniques and medications to clear mucus from the lungs, management and prevention of infections, and proper nutrition. The focus of cystic fibrosis patient's care shifts toward new psychosocial challenges created by longer expected life spans, such patients gaining independence from parents and managing personal relationships. The cystic fibrosis clinics already have substantial momentum toward earlier intervention, such as the preventive measures. The data provide support for that trend.

\section{Acknowledgements}

None.

\section{Conflict of interest}

The author declares no conflict of interest. 